\title{
Mortalidad por cardiopatŕas en menores de un año. Chile, 1988. Orientaciones y desafíos para la década del noventa
}

\author{
Jaime Tapia Z ${ }^{1,2}$; Raúl Zilleruelo B. ${ }^{1}$; Helmut Jaeger $\mathbf{L}^{1}$ \\ Infant's mortality by heart disease in Chile 1988
}

\begin{abstract}
An analysis of infant's deaths caused bv heart diseases in Chile during vear 1988, was performed through a critical review of 5,598 death's certificates and 4,419 infant death audits. The mortality rates due to congenital cardiac defects were 177 per 100,000 liveborns (LB) and varied between 31.5 and 293.0 for 100,000 LB along the country's 26 health districts. In $69 \%$ of death's certificates and $25 \%$ of death s audits, diagnoses vere nonspecific regarding to the type of cardiac malformation, in 15\% of deaths diagnostic orientation was incorrect and in $32 \%$, clinical suspicion of congenital heart disease was raised by the primarv physician but patients did not reach specializ. ed treatment. One half of deaths occurred at general regional non specialized hospitals and $28 \%$ at hospitals of higher complexity. Of these late, $10 \%$ occurred after corrective pr palliative surgery and $7 \%$ in patients considered to be inoperable.

IKey words: congenital heart disease, death rate. mortality, infants.
\end{abstract}

Los métodos de diagnóstico cardjovascular no invasivo y el desarrollo de técnicas quirúrgicas que tienden a correcciones anatómicas $y$ definitivas en pacientes de menor edad y mayor riesgo han modificado el perfil de la población infantil sobreviviente con cardiopatías congénitas en los paises desarrollados durante los últimos veinte años ${ }^{1}$. En Chile, a fines de la década de! ochenta, $20 \%$ de las muertes infantiles ocurrían en niños con anomalías congénítas ${ }^{2}$ y las audito. rías de fallecidos mostraban que $5 \%$ de las defunciones por causas conocidas estaban determinadas por enfermedades del corazón o grandes vasos ${ }^{3}$. Para reducir este riesgo, prácticamente estable desde la década del setenta ${ }^{4-6}$, se ha señalado la necesidad de mejorar la disponibilidad de recursos y de desanollar programas nacionales que aborden con decisión y en profundidad las necesidades de los afectados ${ }^{\text {7-9 }}$.

Este estudio es un esfuerzo por cuantificar el problema de las cardiopatías en los niños a través de su impacto en la mortalidad infantil, identificar algunas de las variables más relevan.

1. Centro Cardiovascular, Hospital Luis Calvo Mackenna.

2. Pediatra Hospital de Chillán. Beca de Cardiologia Infantil, Universialad de Chile (financlada por Laboratorios Recalcine). tes en juego y, por extrapolaciön, seffalar aspectos fundamentales y prioridades que debieran considerarse en las políticas de salud nacionales sobre la materia.

\section{Material y Método}

Se analizaron las defunciones de menores de un ẫo, según residencia, ocurridas entre el 1 de enero y el 31 de diciembre de 1988 , en los 26 servicjos de salud del pais, utilizando como fuentes de datos los certificados de defunción y las auditorías de muertes infantiles. De ias cintas del archivo de la Unidad de Informática del Ministerio de Salud, se revisaron 5.598 certificados de defunción de menores de un año, codificados con cuarto dígito según la causa básica de muerte ${ }^{10}$, consjderando como cardiopatías los códigos $391,420,422$, $425,427,428,745$ y 746. Con esta información se elaboran las tasas de mortalidad infanti. (TMI), por cardiopatías en menores de un âto (TMC) y la relación CI/CE (nímero de defunciones que aparecen como cardiopatía inespecifica -código 746,9- dividido por el número de defunciones con diagnóstico anatómico específico) para cada SS det pajs. Como denominador de las tasas se utilizó la estimación del Ministerio para el número de nacidos vivos en el año $1988^{2}$, el que fue corregido en el nivel provincial con los factores de corrección de H. Gutiérrez" y en el total nacional con $95 \%$ de integridad.

De las auditorias de muertes infantiles (neonatal e infantil tardía) cortespondientes al período de estudio $y$ remitides por los servicios de salud a la unidad de análisis del Ministerio, se revisaron individualmente 
4.419, para seleccionar aquellas en que el defecto cardiovascular aparecía como causa básica, asociada o intercurrente de defunción. De éstas, en $42 \%$ se había confirmado la cardiopatía por necropsia, en $33 \%$ por evaluación clínica cardiológica, en $7 \%$ con procedimientos ecocardiográficos $y$ en $9 \%$ en intervenciones quirúrgicas. Se eliminaron del análisis 33 casos de enfetmedades cromosómicas letales, 36 con malformaciones múltiples severas, anencefalia, hidrocefalia extrema u otras anomalías letales no cardíacas y 18 neonatos con menos de $1,000 \mathrm{~g}$ de peso al nacer, en quiene; el defecto cardiovascular claramente no condicionaba la defunción. Todos los casos con trisomía 21 fueron incluidos. Con la información obtenida de las auditorías de muerte se estudiaron variables como tipo de defecto, edad, estado nutricional, atención médica, manejo médica. quirúrgico, causas asociadas, principales centros de referencia, $e$ infecciones intsahospitalarias. La variable evitabilidad de la defunción se desestimó, por la baja integridad del registro $(63 \%)$ y los distintos criterios que parecen existir para la definición de muerte evitable -por causa cardiovascular - en los distintos servicios de salud del país.

Se definió como "espera carđíológica" el tiempo transcurrido entre la hipótesis clínica inicial y la confirmación por cardjólogo pediátrico. Se denominó "control cardiológico" a la etapa en que el paciente se mantuvo bajo control, en tratamiento médico, después que el diagnóstico habia sido definido -clínicamente o con exámenes, invasivos o no-y el paciente está en tratamiento médico. Se llamó "espera quirúrgica" al espacio de tiempo entre la indicación del tratamiento quirúrgico y su ejecución. Se consideró como defunciones quirúrgicas precoces a las que ocurtieron en los 30 días siguientes a la cirugía y tardías las que sucedieron posteriormente.

\section{Resultados}

Durante el año 1988 se estimaron, en Chile, 296.581 nacidos vivos, de los cuales $40,4 \%$ serían residentes de los 6 servicios de salud de la Región Metropolitana. Según los certificados de defunción, hubo 347 defunciones por enfermedades cardíacas en menores de un atto (321 malformaciones congénitas y 26 afecciones secundarias o adquiridas); pero, según las auditorias de muerte, la cifra fue 357 .

La tasa de mortalidad infantil fue 18,9 por mil nacidos vivos. En la Regtón Metropolitana fluctuó entre 12,3 y 18,5 y en el resto de los servicios de salud del país, entre 7,2 y 24,8 . La tasa de mortalidad por cardiopatias alcanzó un promedio nacional de 117 por cien mil nacidos vivos, fluctuando entre 76,3 y 143,2 en la Región Metropolitana y de 31,5 a 293,0 en los restantes servicios.

La tabla 1 muestra las tasas de mortalidad in. fantil, las de mortalidad por cardiopatias y la relación $\mathrm{CI} / \mathrm{CE}$ por cada servicio de salud. En aquellos donde la TMC era más elevada, la TMI era, por to general, superior al promedio nacional, con excepción del Servicio Metropolitano Oriente, que, con elevada TMC, tenía la segunda TMI más baja del país. Los servicios de salud donde la relación CI/CE era más favorable (servicios $25,26,2,11,14,19,20,21)$ tenían también, por lo general, tasas de mortalidad por cardiopatía bajas, con la excepción de Iquique, donde el riesgo de defunción por cardiopatías en menores de un año es el más alto de Chile (tabla 1).

Tipo de cardiopatia. No se especificaba el tipo anatómico del defecto cardiovascular en $64 \%$ de los certificados de defunción y $25 \%$ de las auditorías de fallecjdos. La tabla 2 muestra la distribución porcentual de las defunciones por distintas cardjopatías en menores de un año, especificando las que ocurren después del tratamiento quirúrgico. Se observó que $8,4 \%$ de los recién nacidos y $10,9 \%$ de los menores de un año que fallecieron por defectos cardiovascula. res recibieron tratamiento quirúrgico, pero, por lo menos, otras 100 defunciones infantiles por cardiopatías susceptibles del beneficio de la cirugía (28\%) no lo alcanzaron.

Edad de la defunción. Veinte niños fallecieron en el primer día de vida $(6 \%)$, sesenta y cinco en la primera semana (18\%) y ciento treinta $y$ uno en el primer mes $(37 \%)$. La figura 1 muestra el número de defunciones según edad (figura 1). En ella se distinguen tres períodos según la velocjdad de ascenso de la curva: el primero - con riesgo de muerte más ałto-se prolongaba desde el nacimiento hasta la tercera semana de vida, en el que se produjeron $35 \%$ de las defunciones el primer año; un periodo intermedio, hasta el término del quinto mes, en que ocurtieron 40\% de las muertes; y el último en el que la pendiente era más lenta. Se indica también en el gráfico la edad promedio de defunción en las principales cardiopatías, siendo la hipoplasia de ventrículo izquierdo (HVI) la más precoz (10 \pm 7 días) y la más tardía el canal auriculoventricular ( $182 \pm 86$ días).

Causas asociadas. En 37\% de los neonatos y $80 \%$ de los lactantes había otras afecciones asociadas a la cardiopatía que pueden haber precipitado la defunción, como las infecciones bacterianas que se encontraron en $50 \%$ de los cardiópatas fallecidos antes del año. En uno de cada tres de estos pacientes se registró el diag- 
Tabla 1

Tasa de mortaljad infantil (TMI), por cardiopatias en menores (TMC) $y$ relación $(\mathrm{Cl} / \mathrm{CE})$, según servicios de salud. Chile, 1988

\begin{tabular}{|c|c|c|c|}
\hline Servicios de salud & TM[ & TMC & $\mathrm{CI} / \mathrm{CE}$ \\
\hline 1. Arica & 14,2 & 51 & 2,0 \\
\hline lquique & 18,9 & 293 & 1,2 \\
\hline Antofagasta & 17,8 & 120 & 3,0 \\
\hline 4. Atacama & 23,4 & 93 & \\
\hline Coquirnbo & 18,9 & 172 & 2,5 \\
\hline Valparaíso & 19,0 & 132 & $\mathbf{3}, \mathbf{0}$ \\
\hline Viña-Quillota & 18,9 & 154 & 1,5 \\
\hline San Felipe-Los Ander & 18,4 & 138 & 2,0 \\
\hline Metropolitano Norte & 16,7 & 91 & 1,2 \\
\hline Metjopolitano Occidente & 18,5 & 83 & 1,1 \\
\hline Metropolitano Central & 13,7 & 95 & 0,9 \\
\hline Metropolitano Oriente & 12,3 & 143 & 1,2 \\
\hline Metropolitano Sur & 15,9 & 76 & 1,7 \\
\hline Metropolitano Sur-Oriente & 15,3 & 114 & 0,9 \\
\hline Libertador B. O'Higgins & 17,6 & 120 & 5,3 \\
\hline Maule & 21,0 & 90 & 3,5 \\
\hline N̂uble & 22,6 & 119 & 2,7 \\
\hline Concepción-Arauco & 24,8 & 185 & 2,5 \\
\hline Talcahuano & 17,1 & 77 & 1,0 \\
\hline Bío-Bio & 20,0 & 115 & 1,0 \\
\hline Araucania & 21,4 & 72 & 1,0 \\
\hline Valdivia & 20,3 & 98 & 3,0 \\
\hline Osorno & 15,6 & 100 & 5,0 \\
\hline Llanchipal & 22,4 & 138 & 5,5 \\
\hline Aysén & 24,6 & 9] & 0,0 \\
\hline 26. Magallanes & 7,2 & 32 & 0,0 \\
\hline Total pais & 18,9 & 117 & 1,8 \\
\hline
\end{tabular}

TMJ: por mil nacidos vivos (provisoria).

TMC: por cien mil nacidos vjyos.

nóstico de bronconeumonia. El estado de nutrición era normal en $48 \%$ de los fallecidos y en $32 \%$ se trataba de desnutridos moderados o severos según Semps ${ }^{\text {t2 }}$. En $25 \%$ de las defunciones de cardiópatas con desnutrición grado 2 y $42 \%$ de las con grado 3 habia trisomía 21. Las infecciones nosocomiales se notificaron en $14 \%$ de las defunciones intrahospitalarias por cardiopatia. Las tasas fluctuaron entre $20 \%$ y $7,6 \%$ en los servicios de salud que notificaron el hecho.

Atención médica. A pesar que más de $98 \%$ de los niños menores de un año con cardiopatías, fallecidos, estaba bajo control médico, $25 \%$ de las muertes en neonatos y $11 \%$ de las infantiles tardías ocurrieron sin que se sospechara clínicamente el defecto casdiovascular. Durante la espera cardiológica ocurrieron $46 \%$ de las muertes neonatales y $21 \%$ de las de niños mayores de un mes, bajo control cardiológico 30\% de las infantiles tardias, mientras en espera de cirugia sucedieron $4 \%$ de las neonatales y $10 \%$ de las de lactantes. El porcentaje de pacientes desahuciados de todo tratamiento quirúrgico alcanzó a $12 \%$ de los recién nacidos y $5 \%$ de los mayores de un mes, pero los criterios difirieron objetjva y sustancialnente, iñcluso entre los distintos centros cardiológicos del país. La letaljdad quirúrgica estuvo representada por $8 \%$ de los neonatos y $12 \%$ de los lactantes fallecidos por cardiopatía antes del año. Hubo sejs muertes operatorias tardías, sólo una de ellas por falla cardiovascular cuatro meses despues de valvulotomía y operación de Blalock en un paciente con atresia pulmonar. De las muertes postoperatorias, $88 \%$ ocurrieron en pacientes intervenidos en el Hospital Lujs Calvo Mackenna, del Servicio de Salud Metropolitano Oriente, y $9 \%$ en hospitales de la $V$ Región. Tres tipos especificos de malformaciones cardiacas congénitas (transposición de grandes arterias, drenaje venoso pulmo. nar anómalo total y tetralogía de Fallot) representaron $91 \%$ de las muertes quirúrgicas neonatales y $56 \%$ de las ocurridas en menores de un año (tabla 2). 


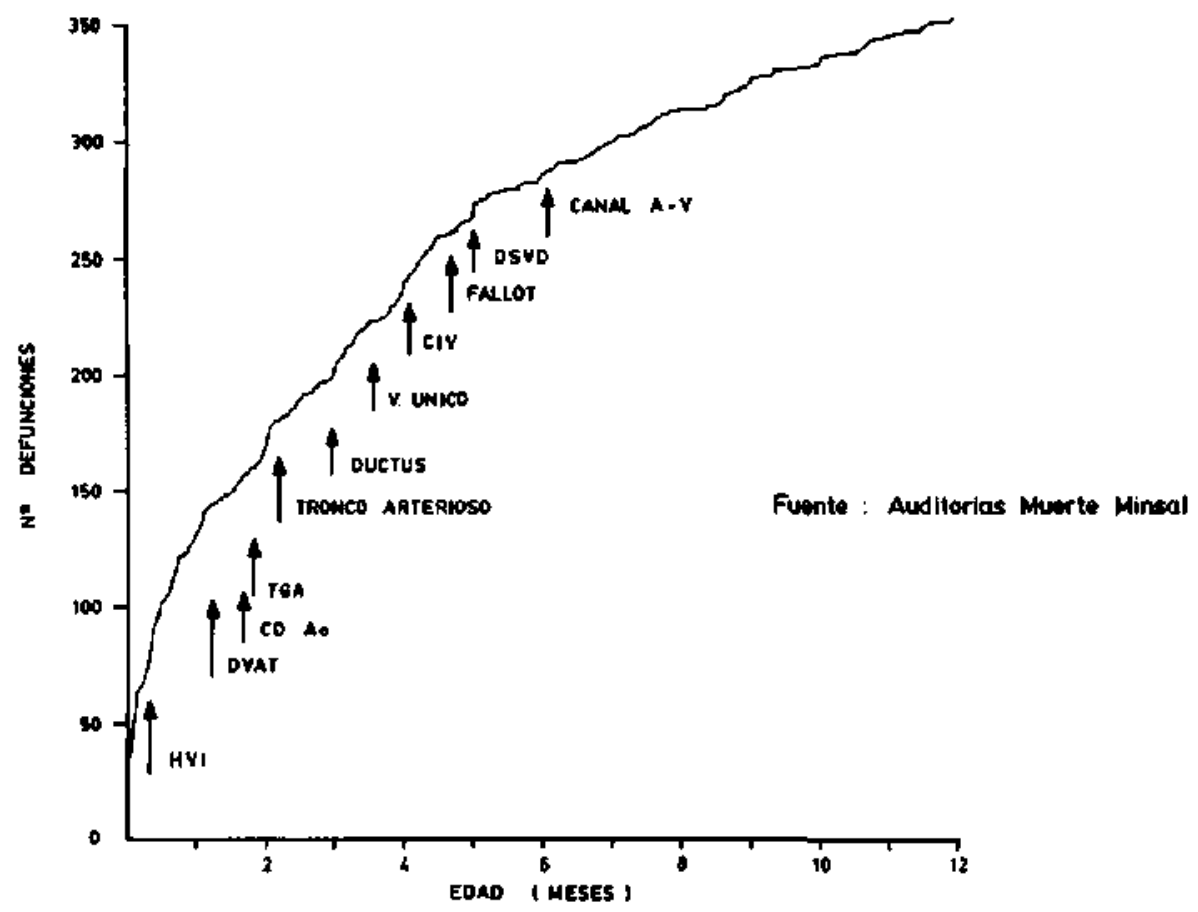

Fig. 1: Número de defunciones infantiles por cardiopatías y edad promedio de muerte en las principales de ellas. Chile, 1988. HVI: hipoplasía ventrículo izquierdo; DVAT: drenaje venoso anómalo total; CoAo: coartación de la aorta; TGA: transposición grandes vasos; DSVD: doble salida ventrículo derecho; CANAL A-V: canal aurículo ventricular; V. UNICO: ventriculo único.

Tabla 2

Defunciones por cardiopatías en menores de un afio. Chile, 1988

\begin{tabular}{|c|c|c|c|c|c|c|}
\hline \multirow[t]{2}{*}{ Tipo defecto anatómico } & \multicolumn{3}{|c|}{ Defunciones } & \multicolumn{3}{|c|}{ Postcirugía } \\
\hline & RN & Total & $\%$ & RN & Total & $\%$ \\
\hline No especificado & 38 & 91 & 25,5 & & & \\
\hline Comunicación interventrłcular & 12 & 64 & 17,9 & - & 1 & 2,6 \\
\hline Drenaje venoso pulmonar anómalo total & 13 & 19 & 5,3 & 5 & 7 & 17,9 \\
\hline Transposición grandes arterias & 9 & 18 & 5,0 & 4 & 9 & 23,1 \\
\hline Tronco arterioso & 9 & 18 & 5,0 & - & 4 & 10,2 \\
\hline Canal auriculoventricular & 2 & 18 & 5,0 & - & 2 & 5,1 \\
\hline Tetralogia de Fallot & 3 & 15 & 4,2 & 1 & 6 & 15.4 \\
\hline Ventrículo único & 4 & 13 & $3, ?$ & - & 2 & 5,1 \\
\hline Coartación e hipoplasia aórtica & 4 & 15 & 4,2 & - & 1 & 2,6 \\
\hline Hipoplasia V.D. y atresia A.P. & 2 & 9 & 2,5 & - & 3 & 7.7 \\
\hline Doble salida de ventrículo derecho & 2 & 4 & 1,1 & 1 & 3 & 7,7 \\
\hline Estenosis aórtica & 2 & 4 & 1,1 & - & 1 & 2,6 \\
\hline Atresia tricuspidea & 1 & 3 & 0,8 & & & \\
\hline Comunicación interauricular & 1 & 5 & 1,4 & & & \\
\hline Ductus & 5 & 20 & 5,6 & & & \\
\hline Hipoplasia de ventrículo izquierdo & 19 & 19 & 5,3 & & & \\
\hline Miocardiopatías & - & 10 & 2,8 & & & \\
\hline Estenosis mitral & - & 2 & 0,6 & & & \\
\hline Enfermedad de Kawasaki & - & 2 & 0.6 & & & \\
\hline Enfermedad de Ebstein & 2 & 2 & 0,6 & & & \\
\hline Otcas & 3 & 6 & 1,7 & & & \\
\hline Total & 131 & 357 & 100,0 & 11 & 39 & 100,0 \\
\hline
\end{tabular}

Fuente: auditorias de muertes infantiles. 
Niveles de atención. Menos de $5 \%$ de los niños con cardiopatías fallecieron sin haber consultado en las 72 horas previas a la defunción, 53\% murieron en hospitales base de los servicios de salud, $27 \%$ en centros de referencia cardiovascular y $20 \%$ en hospitales periféricos. En los hospitales base de los servicios, generalmente calificados en las dos categorías superiores del país en dotación y recursos, 19\% de los niños que fallecieron con afecciones cardiacas murieron antes de transcurridas $\mathbf{4 8}$ horas desde el ingreso, $64 \%$ no fueron derivados, a pesar de hospitalizaciones más largas que dicho lapso de tiempo, y en $9 \%$ el deceso ocurrió sin que se diagnosticara su cardiopatia. La proporción de pacientes desahuciados de cirugía alcanzó en estos hospitales a $8 \%$. En los centros de referencia cardiotascular, $45 \%$ de las muertes por cardiopatías ocurrieron bajo control cardiológico ambulatorio, 33\% después de cirugia cardiovascular, 13\% antes de transcurrir 48 horas desde el ingreso, $6 \%$ en niños desahuciados para la cirugía $y$ sólo $3 \%$ en espera de tratamiento quirúrgico.

En la figura 2 se representa el númexo de defunciones en pacientes con cardiopatías según el servicio de salud correspondiente a su residencia (parte superior) y el número de las mismas que ocurrieron en centros de referencia cardiovascular. Se puede observar que para casi todos los servicios de salud predominaron las defunciones de cardiópatas no derivados o calificados en la categoría otros, que incluye principalmente las que sucedieron antes de 48 horas después de ingresar. Setenta y tres por ciento de las derivaciones de estos pacientes fueron hechas al centro cardiovascular del Hospital Luis Calvo Mackennt, en 1988, y $11 \%$ al Hospital Roberto del Río, como también se aprecia en la figura 2.

\section{Discusión}

Estudios recientes sefialan que en Chile la incidencia de niffos con malformaciones es 6,2\% entre los mortinatos del Area Norte de Santiago y que 13,7 por mil NV tienen anomalías cardjacas ${ }^{13,14}$. Según estimaciones internacionales, durante la última década, la incidencia de malformaciones cardíacas congénitas habría aumentado de 5 a $?$ por mil NV y la de anomalías que requieren intervención cardiovascular en la infancia se habria mantenido estable en 3,5 por mil nacjdos vivos'. De acuerdo a estas cifras, la demanda de atención por malformaciones cardíacas creceria anualmente en Chile 2.000 a 4.000 casos nuevos y alrededor de 1.000 nuevos pacientes en estado crítico requerirían cirugía precoz. Coincidentemente, en los últimos afios, se ha observado aumento de la frecuencia de diagnósticos de cardiopatías y otras malformaciones en los egresos hospitalarios ${ }^{15}$. En el tercer trimestre de 1989, por ejemplo, 62\% de los días-cama ocupados y $50 \%$ de los egresos del Hospital Luis Calvo Mackenna correspondían a pacientes con anomalías congénitas.

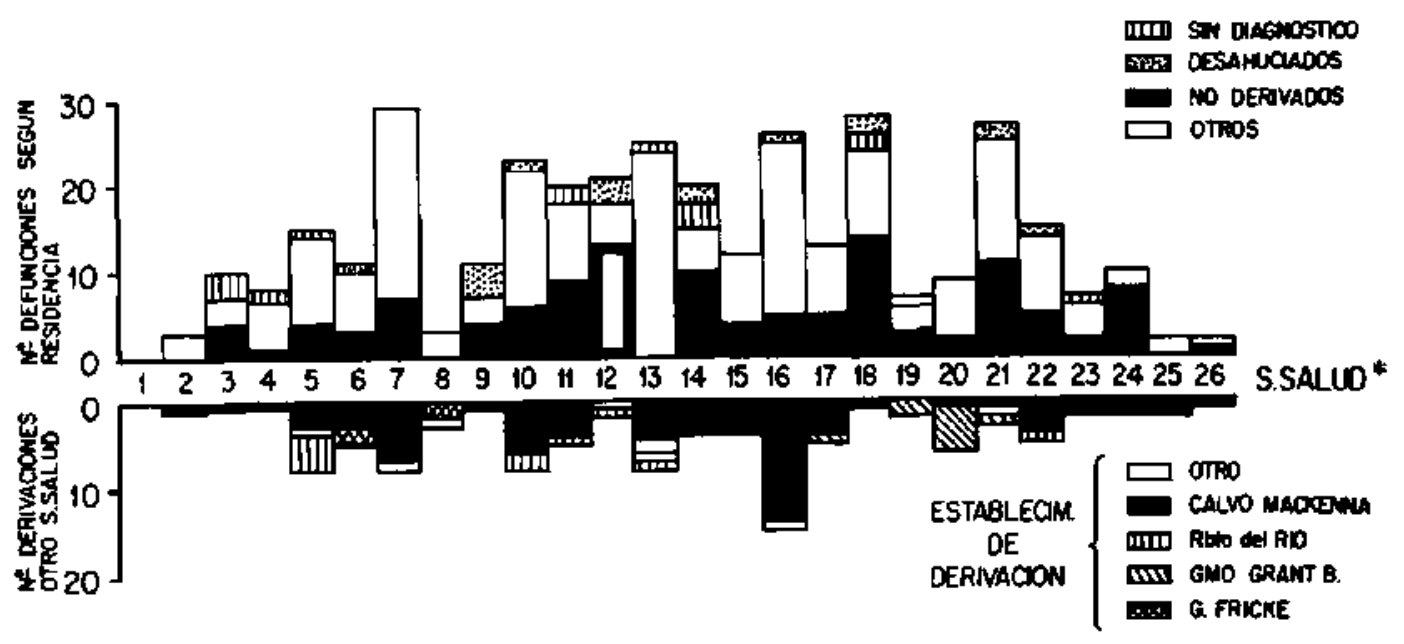

Fig. 2: Número de defunciones infantiles por cardiopatía en los servicios de salud y centros de referencia cardiovacular, según residencia. Chile, 1988. (*El orden numérico de los Servicios de Salud corresponde a la tabla 1). 
De acuerdo a nuestros resultados - si la declaración de domicilio en Jas auditorías de muerte es correcta- existiría una fuerte omisión en la estimación de la TMC del pais, ya que aplicando a ésta el mismo porcentaje de sobremortalidad que se observa entre la TMI nacional y la del Servicio de Salud Metropolitano Oriente, la tasa de mortalidad por cardiopatías debería ser cercana a 220 por 100 mil NV, casi el doble de la oficial.

Esta enorme brecha, entre la magnitud real y la oficial del riesgo de muerte infantil por enfermedades del aparato cardiovascular en el país, denota el desarrollo insuficiente de la capacidad para hacer el diagnóstico clínico, no invasivo, en la mayoría de sus servicios de salud, la poca frecuencia con que se realizan necropsias en las defunciones de menores de un año y, probablemente, posibilidades insuficientes de acceso a los centros terciarios de atención. Corroboran esta apreciación el elevado número de cardiópatas que fallecieron sin diagnóstico o sin que se definiera el tipo de defecto anatómico, las diferencias en las tasas de mortalidad por cardiopatías entre las regiones y la gran heterogeneidad regional en la relación CL/CE, que varió de 0 a 5,5 en los distintos servicios de salud.

El primer problema práctico para una política de salud destinada a abordar esta realidad es una adecuada asignación de recursos ${ }^{7,8}$, que debe apoyarse sobre todo en principios éticos que protejan el derecho de los menos favorecidos a una atención equitativa ${ }^{16}$, evitando la duplicación innecesaria de equipos de alto costo ${ }^{7}$, pero al mismo tiempo tendiente a homologar, a lo largo del país, las oportunidades de acceso geográfico y económico a los beneficios de los centros cardiovasculares, asi como a favorecer el flujo expedito de los pacientes, entre los distintos niveles de atención médica.

De acuerdo al consenso logrado en el último Congreso Latinoamericano de Cardiología se necesitaría un centro cardiovascular pediátrico de nivel terciario por cada 5 millones de habitantes. En Chile existe uno solo: el del Hospital Luis Calvo Mackenna, en el sector Metropolitano Oriente, que durante la década del ochenta tuvo un promedio anual de 5.543 consultas am. bulatorias; 442 intervenciones mayores (381$503) ; 185$ con circulación extracorpórea (140224) y 320 estudios hemodinámicos. En los últimos affos otros dos hospitales de Santiago y uno de la V Región han inicjado el tratamiento quirúrgico de algunos tipos de cardiopatías congénitas. En la prôxima década el rivel central deberá estimular un crecimiento ordena. do y racional de otros centros de atención de la especialidad, con mejor definición de Jos roles de cada cual, de acuerdo a la estratificación y regionalización de los servicios de salud, dotando adecuadamente, con recursos humanos y tecnológicos, a aquellos que con medios limitados han dernostrado más eficiencia en el manejo de los niños con cardiopatias, concentrando en ellos los procedimientos de mayor complejidad y las técnicas quirúrgicas que demandan más gastos y esfuerzos técricos, otorgando, a la vez, el nivel más bajo de riesgo y los beneficios de la experiencia clínica mejor asentada ${ }^{16-30}$.

El segundo objetivo debiera ser la formación integral de cardiólogos pediatzas y cardiocirujanos infantiles, la utilización más eficiente del djagnóstico no invasivo y la introducción en mayor escala a nivel nacional de medicamentos como la prostaglandina $\mathrm{E}$ y la indometacina endovenosa, tendiendo a dar mejor cobertura diagnóstica y terapéutica en centros regionales de nivel secundario a las afecciones cardiovasculares médico-quirúrgicas menos complejas, poniendo énfasis en el recién nacido con sospecha de cardiopatía.

Un tercer desafio, tal vez el más grande, sería la utilización de la red de atención primaria para el control de salud y seguimiento de los niños afectados por cardiopatía con un enfoque de riesgo ${ }^{21}$, ya que, pese a no mostrar compromiso hemodinámico evidente, los lactantes en esta situación suelen deteriorarse y fallecer por mobilidad asociada, particularmente bacteriana $y$ nutricional. Esto supone la elaboración de un plan nacional de manejo de estos nifros que evalúe correctamente los factores de riesgo, proponiendo estrategias regionales para prevenir, detectar y tratar complicaciones, derivar oportuna $y$ eficazmente los enfermos al nivel de atención médica que corresponda, reduciendo el alto porcentaje de defuncjones que ocurren precozmente al ingreso en los hospitales, la congestión de los hespitales base y el traslado no oportuno a los centros de atención terciaria.

La mejor definición de algunos aspectos de la especjalidad, como los relativos a la prevención y el diagnóstico prenatal de cardiopatías incompatibles con la vida, aberraciones cromosómicas y otras embriopatias, como la alcohóli$\mathrm{ca}^{22-26}$ y del manejo de cardiopatías comple. 
jas, para los cuales no existe aún tratamiento quirúrgico exitoso, permitirá mejorar las opciones de un número no despreciable de enfermos cardiovasculares de elevada letalidad.

\section{Resumen}

Se analizaron las muertes de menores de un año ocurridas en Chile durante 1988 , revisando 5.598 certificados de defunción y 4.419 auditorías de fallecidos. La tasa de mortalidad por cardjopatías en el país fue 117 por cien mil nacidos vivos y varió entre 31,5 y 293,0 en los diferentes servicios de salud. En $69 \%$ de los certificados y $25 \%$ de las auditorías no se especificaba el tipo anatómico del defecto cardjovascular, en $15 \%$ de los nifros fallecidos por causas cardiovasculares no se había hecho el diagnóstico clínico y $32 \%$ no fueron evaluados por un cardiólogo. La mitad de las muertes ocurrieron en hospitales base (tipo I) y $28 \%$ en centros de atención terciaria ( $10 \%$ después de cirugía paliativa o correctora y $7 \%$ considerados inoperables). Parece necesario tomar, en esta década, medidas para mejorar los flujos de derivación de pacientes, extender el empleo de los métodos de diagnóstico no invasivo y de ciertos procedimientos farmacológicos, como el uso de prostaglandinas e indometacina, $y$ distribuir los recursos de acuerdo con una planificación nacional tendiente a mejorar la definición de los niveles de atención médica y estratificación de los servicios de salud.

(Palabras clave: malformaciones cardíacas, mortalidad.)

\section{Agradecimientos}

A Cesar Gattini, Carlos Reyes y Dina Zurita, por el acceso a la información básica e importantes sugerencias metodológicas. A Victoria Llanos y Gladys Salas, por la transcripción de los borradores.

\section{Referencias}

1. Woodrow Benson, J.: Changing profile of congenital heart disease. Pediatrics 1989; 83: 790-91.

2. Ministerio de Salud: Indicadores biodemográficos, 1989.

3. Ministerio de Solud: Defunciones infantiles y fetales tardías según el sistema de auditoría de muertes, 1987. Depto. Coordinación, Unidad de Análisis, 1989.
4. Tapia Z., J.; Corrasco, M.A.: Niveles y tendencias de la mortalidad por enfermedades y anomalías congénitas del aparato circulatorio en la niñez. Chile, 1968-1982. Boletín de Cardiología (Chile), 1984; 3: 91-98.

5. Kirschbaum, A.; Páez, G.: Anomalias Congénitas. Chile, 1982. Resumen V Jornadas Chilenas de Salud Pública. 1985.

6. Blanco, R.; Kamps, J.; Palomino, H.: Defunciones por malformaciones congénitas en Chile, 197079. Rev Med Chil 1983;111: 510-18.

7. Toro. A.C.: Atención cardiovascular para niños: se necesitan recursos. Rev Chil Pediatr 1986; 57: 391-92.

8. Farrí, A.O.: Cardiología Pediátrica. Pedjatría $1978 ; 21: 3$.

9. Jaeger, L.H.: Reseña histórica, en Artaza, O.; Zilleruelo, R.: Manual de Cardiología y Cirugía Cardiovascular Infanti. Ed. Mediterráneo, Santiago, Chile, 1989.

10. OPS-OMS: Manual de la Clasificación Estadística Internacional de enfermedades, traumatisnos $y$ causas de muerte, 1978. Vol 1, Publicación Científica 353, Washington, D.C. 20036. EUA.

11. Gutierrez, H.: Integridad del registro de nacidos vivos en Chile, 1953-66. Depto. Bioestadística U. de Chile, 1968.

12. Sempe, M.; Roy, P.; Pedrón, G.: (París, 1971) en Ministerio de Salud. Normas de Pediatría, Chile, 1976.

13. Nozer, H.J.; Cifuentes, O.L.; Catalä. M.J.: Incidencia de malformacjones congénitas en mortinatos. Rev Chil Pediatr 1989; 60: 208-1 l.

14. Philippi, R.O.; Rencoret, S.G.; Pinto, S.M.: Incidencia de cardiopatías congénitas en nacidos vjvos. Rey Chil Pediatr 1986; 57 : 447-51.

15. Urriola, B.P.; Mulhausen, M.G.: Malformaciones cardíacas en recién nacidos hospitalizados. Rev Chil Pediatr 1986; 57 : 422-25.

16. Duggan, J.M.i Resource allocation and biocthics. Lancet, 1989; 1(8641): 772-73.

17. Alcaide, A.; Zilleruelo, R.; Eimbcke, F, et al.: Incidencia de cardiopatías congènitas en las autopsias de los últimos 10 años, 1955-64. Rev Chil Pediatr 1965;36: 698-706.

18. Vicufa, W.D.; Eimbcke, M.F.; Zilleruelo, B.R.: Valvuloplastia pulmonar percutánea con balón. Boletín de Cardiología (Chile), 1988; 7: 123-129.

19. Palominos, G; Piwonka, G.: Franck, R.: Bem, L.; Edwerds, A.: Cirugía de las cardiopatías congénitas en el Hospital Clínico de la Universidad de Chile. Cardiología Clínica, 1989;6: 10-14.

20. Artaza, $O$; Gómez, $O$; Arretz, $C$ et al: Resultados de ta reparación quirúrgica del canal auriculoventricular completo. Revista Chilena de Cardiología, 1990; 9: 157-162.

21. Sanué, P.E.; Bertoni, N. V.; Gonzalo, D.A.; Sertano, $V$. El concepto de riesgo y el cuidado de la salud, Pub Cient CLAP 1007, Montevideo, Uruguay, 1984.

22. Medino, E.: Una poljtica de prevención de enfermedades cardiovasculares. Boletín Hospital S.J. de Dios, 1986: 36-34. 
23. Fami, A.O.: Prevención de las cardiopatías congé. nitas. Pediatría (Santiago), 1978; 21:5-8.

24. Menn, R.M.; Pacheco, C.V.; Lama, T.A.: Malformaciones cardíacas congénitas en el Síndrome Alcohólico Fetal. Rev Chil Pedjatr 1986; 57 : $398-400$.
25. Strong. W.B.: Dennison. A.: Pediatric Proventive Cardiology. Atherasclerosis and Coronary Heart Disease. Pediatr Rev 1988; 9: 303-13.

26. Dewcher, Z.: Cardiopatías Congénitas; orjentación y tratamiento en el primer año de vida. Boletín de Cardiología (Chile) 1985; 4: 17-23.

Esta publicación está disponible en copias de microfilms de 16 y $35 \mathrm{~mm}$ y microfichas de $105 \mathrm{~mm}$, las que pueden solicitarse a:

University Microfilms International

300 North Zeeb Road

Ann Arbor, Michigan 48106, USA.

This journal is also available in $16 \mathrm{~mm}$ microfilm, $35 \mathrm{~mm}$ microfilm and $105 \mathrm{~mm}$ microfilm copies through University Microfilms International, 300 North Zeeb Road, Ann Arbor, Michigan 48106, USA. 